\title{
Trajectory tracking control with preview action for a class of continuous-time Lur'e-type nonlinear systems
}

\section{Xiao Yu' ${ }^{1 *}$ (1) and $\mathrm{Li} \mathrm{Li}^{2}$}

"Correspondence:

yuxiao19@sdjzu.edu.cn

'School of Science, Shandong

Jianzhu University, Jinan, China

Full list of author information is

available at the end of the article

\section{Springer}

\begin{abstract}
A novel preview control scheme is developed for the trajectory tracking problem of continuous-time Lur'e-type nonlinear systems. With the aid of the translation approach, the state augmentation technique along with some special mathematical manipulations, an augmented error system including preview information is constructed. The tracking control problem is thereby reduced to a standard $H_{\infty}$ controller design problem. Meanwhile, the integrator is introduced to eliminate the steady-state tracking error. The controller design condition is established in terms of a linear matrix inequality (LMI). As for the original system, the tracking controller is a state feedback controller with tracking error integral plus preview action. Finally, an example is given to illustrate the tracking performance of the proposed method.
\end{abstract}

Keywords: Nonlinear Lur'e-type system; Tracking control; Preview control; LMI

\section{Introduction}

Preview control is an important control method, which is capable of effectively improving the tracking performance of the closed-loop system by means of the available future information about the reference signal and/or the disturbance $[1,2]$. Because of this, preview control is widely used in trajectory tracking problem of control systems. The original studies in this field can be traced back to the work in [3-5] where the concept and models of preview control were discussed thoroughly, and some potential applications were provided. For the deterministic and stochastic linear time-invariant systems in both continuous-time and discrete-time domain, the classical Riccati equation approach was extensively applied to optimal preview controller design based on linear quadratic optimal control theory [6-13]. Particularly, the authors in [14-16] proposed a new preview control method for discrete-time systems by applying information fusion estimation theory. Subsequently, the popular Riccati equation approach was further generalized to the preview control for linear descriptor systems in [17-21]. Additionally, combining with robust control theory, the preview control problems were well characterized in [22-26] for linear uncertain systems and special nonlinear systems using LMI approach. Unfortunately, most of the aforementioned studies concern linear systems, whereas very few

(c) The Author(s) 2020. This article is licensed under a Creative Commons Attribution 4.0 International License, which permits use sharing, adaptation, distribution and reproduction in any medium or format, as long as you give appropriate credit to the original author(s) and the source, provide a link to the Creative Commons licence, and indicate if changes were made. The images or other third party material in this article are included in the article's Creative Commons licence, unless indicated otherwise in a credit line to the material. If material is not included in the article's Creative Commons licence and your intended use is not permitted by statutory regulation or exceeds the permitted use, you will need to obtain permission directly from the copyright holder. To view a copy of this licence, visit http://creativecommons.org/licenses/by/4.0/. 
results were developed for nonlinear control systems, especially in the continuous-time domain. Therefore, there is still enough room to expand the applicability scope of preview control.

Many nonlinear physical systems such as Wiener model, $\mathrm{n}$-scroll attractors and Chua's circuit can be represented as a feedback connection of a linear dynamical system and a nonlinear element satisfying a certain sector constraint. Such systems are often referred to as Lur'e-type systems $[27,28]$. In the literature, there has been considerable work dealing with the trajectory tracking control problem of Lur'e-type nonlinear systems owing to their extensive practical applications. In [29-31], the absolute tracking control of Lur'etype systems was considered and the algebraic conditions for controller design were systematically discussed. Taking the faults of both actuators and sensors into account, a reliable tracking control scheme was provided for continuous-time Lur'e-type descriptor systems in [32]. In [33], a high-gain fractional-order control scheme was proposed for Lur'e-type system with unknown disturbances to accurately tracks the desired reference signal. In [34], the tracking problem of discrete-time Lur'e-type systems was handled via output feedback model prediction control. Beyond that, many interesting results concerning some special trajectory tracking problems for this class of nonlinear systems have been obtained in the literature, such as consensus tracking control $[35,36]$ and synchronization control [37-39]. However, how to achieve fine trajectory tracking in the sense that the desired reference signal is previewable has been an open issue hitherto.

Inspired by the above statements, this paper deals with the trajectory tracking control problem of continuous-time Lur'e-type nonlinear systems via preview control for the first time. Compared to the discrete-time counterpart, the preview controller design for continuous-time nonlinear Lur'e-type systems is more challenging. This is mainly because the previewable reference information cannot be efficiently imported into the usual error system through state augmentation. To overcome this obstacle, we first introduce a novel translation approach to deriving the error system. Then, according to the specific characteristics of the state matrix in error system, the preview compensation term based on future reference trajectory could be skillfully attached to the integrator through some mild mathematical manipulations, which is the main contribution of the paper. In this way, the required augmented error system including integral action and preview information is successfully constructed, which transforms the original trajectory tracking control problem into an $H_{\infty}$ controller design problem. This handling procedure provides a new control scheme for trajectory tracking problem of continuous-time nonlinear Lur'e-type systems, thereby effectively expanding the applicable scope of preview control technique.

Notations $R^{n}$ denotes the $n$-dimensional Euclidean space; $R^{n \times m}$ denotes the $n \times m$ matrix space; $P>0(P<0)$ means that $P$ is a real symmetric positive (or negative) matrix; $P>Q$ stands for $P-Q>0 ; I$ and 0 are used to represent the identity matrix and the zero matrix with appropriate dimension, respectively; $L_{2}$ refers to the space of square integrable functions, and for any $\omega(t) \in L_{2}$, its norm is given by $\|\omega(t)\|_{2}=\sqrt{\int_{0}^{\infty} \omega^{T}(t) \omega(t) d t}<\infty$.

\section{Problem formulation}

Consider the following continuous-time nonlinear system:

$$
\left\{\begin{array}{l}
\dot{x}(t)=A x(t)+B u(t)+D f(y(t))+E \omega(t), \\
y(t)=C x(t),
\end{array}\right.
$$


where $x(t) \in R^{n}$ is the state vector, $u(t) \in R^{m}$ is the control input vector, $y(t) \in R^{p}$ is the output vector, $\omega(t) \in R^{q}$ is an external disturbance vector. $A, B, C, D, E$ are known constant matrices of appropriate dimensions. $f(y) \in R^{p}$ is a memoryless time-invariant nonlinearity.

The following assumptions are required for system (1).

A1 The nonlinearity $f(\cdot)$ is in the form of

$$
f(y)=\left[\begin{array}{llll}
f_{1}\left(y_{1}\right) & f_{2}\left(y_{2}\right) & \cdots & f_{p}\left(y_{p}\right)
\end{array}\right]^{T},
$$

where $f_{i}(0)=0$, and there exist constants $\underline{k}_{i}, \bar{k}_{i} \in R$ and $\underline{k}_{i}<\bar{k}_{i}$ such that

$$
\underline{k}_{i} \leq \frac{f_{i}\left(s_{2}\right)-f_{i}\left(s_{1}\right)}{s_{2}-s_{1}} \leq \bar{k}_{i}, \quad \forall s_{1} \neq s_{2}, i=1,2, \ldots, p .
$$

Remark 1 The class of nonlinear systems verifying Assumption A1 covers a wide range of physical systems in the real world, such as robots and Chua' circuits [40]. As commented in $[37-39,41]$, the function $f_{i}(\cdot)$ belongs to the sector $\left[\underline{k}_{i}, \bar{k}_{i}\right]$. Also, the constants $\underline{k}_{i}, \bar{k}_{i}$ in (2) are allowed to be positive, negative or zero, therefore the assumption on the nonlinear terms in this paper is very general. Furthermore, both qualitative analysis and control synthesis for systems with Assumption A1 have been intensively studied in [30-40].

A2 The external disturbance $\omega(t)$ converges to a constant vector $\omega$ as $t \rightarrow \infty$, i.e., $\lim _{t \rightarrow \infty} \omega(t)=\omega$. Also, the difference vector between the disturbance and its limit belongs to $L_{2}$, i.e., $\omega(t)-\omega \in L_{2}$.

Although Assumption A2 seems to be a little strict, in the existing studies and practical applications, there are many disturbance signals satisfying A2; among them, the energybounded disturbance in [37] is a typical example.

A3 The rank of the augmented matrix $\left[\begin{array}{ll}A & B \\ C & 0\end{array}\right]$ equals $n+p$.

The desired reference signal $r(t) \in R^{p}$ is piecewise differentiable with finite discontinuity points in $[0, \infty)$ and satisfies the following assumption:

A4 The reference signal $r(t)$ converges to a constant vector $r$ as $t \rightarrow \infty$, i.e., $\lim _{t \rightarrow \infty} r(t)=r$. Also, the difference vector between the reference signal and its limit belongs to $L_{2}$, i.e., $r(t)-r \in L_{2}$. Furthermore, the reference signal $r(t)$ is assumed to be previewable in the sense that the future values $r(\tau)\left(t \leq \tau \leq t+l_{r}\right)$ are available at each time $t$. $l_{r}$ is called the preview length of the reference signal.

Remark 2 Assumption A4 is a quite standard hypothesis in the field of preview control, which shows that some future information about reference signal can be measured in advance. A lot of studies confirm that inserting such available information into control signal helps to improve the overall tracking performance of the control system tremendously (see $[1-4,6-9]$ and the references therein).

Let the tracking error be

$$
e(t)=y(t)-r(t)
$$


The purpose of this paper is to design a tracking controller with preview action for system (1) such that the output vector $y(t)$ asymptotically tracks the desired reference signal $r(t)$, that is,

$$
\lim _{t \rightarrow \infty} e(t)=\lim _{t \rightarrow \infty}(y(t)-r(t))=0
$$

Remark 3 Robust stabilization problems of Lur'e-type nonlinear systems have been studied in $[27,28]$. The trajectory tracking control for this class of systems has been considered in [30-36]. Nevertheless, there is almost no research work investigating the trajectory tracking problem of Lur'e-type systems via the preview control approach, especially in the continuous-time domain.

Lemma 1 (Schur complement lemma [25]) The symmetric matrix $\left[\begin{array}{ll}S_{11} & S_{12} \\ S_{12}^{T} & S_{22}\end{array}\right]<0$ if and only if one of the following two conditions is satisfied:

(i) $S_{11}<0, S_{22}-S_{12}^{T} S_{11}^{-1} S_{12}<0$;

(ii) $S_{22}<0, S_{11}-S_{12} S_{22}^{-1} S_{12}^{T}<0$.

\section{Derivation of the augmented error system}

In fact, under Assumptions A2 and A4, if the closed-loop system of system (1) can track the reference signal $r(t)$ asymptotically, then there exist steady-state values $x(\infty)$ and $u(\infty)$ such that

$$
\left\{\begin{array}{l}
0=A x(\infty)+B u(\infty)+D f(r)+E \omega \\
r=C x(\infty)
\end{array}\right.
$$

that is,

$$
\left[\begin{array}{ll}
A & B \\
C & 0
\end{array}\right]\left[\begin{array}{l}
x(\infty) \\
u(\infty)
\end{array}\right]=\left[\begin{array}{c}
-D f(r)-E \omega \\
r
\end{array}\right] .
$$

When Assumption A3 holds, the nonhomogeneous linear equation (5) has solutions $x(\infty)$, $u(\infty)$.

Define the new variables

$$
\left\{\begin{array}{l}
\tilde{x}(t)=x(t)-x(\infty) \\
\tilde{u}(t)=u(t)-u(\infty), \\
\tilde{y}(t)=y(t)-r \\
\tilde{r}(t)=r(t)-r \\
\tilde{\omega}(t)=\omega(t)-\omega .
\end{array}\right.
$$

From Eqs. (1), (4) and (6), we get the following dynamics:

$$
\left\{\begin{array}{l}
\dot{\tilde{x}}(t)=A \tilde{x}(t)+B \tilde{u}(t)+D \tilde{f}(\tilde{y}(t))+E \tilde{\omega}(t), \\
\tilde{y}(t)=C \tilde{x}(t)
\end{array}\right.
$$

where $\tilde{f}(\tilde{y}(t))=f(y(t))-f(r)$. 
From $\tilde{y}_{i}=y_{i}-r_{i}$ and $\tilde{f}_{i}\left(\tilde{y}_{i}\right)=f_{i}\left(y_{i}\right)-f_{i}\left(r_{i}\right)$, and combining with Assumption A1, the following inequality is satisfied:

$$
\left(\tilde{f}_{i}\left(\tilde{y}_{i}\right)-\underline{k}_{i} \tilde{y}_{i}\right)\left(\tilde{f}_{i}\left(\tilde{y}_{i}\right)-\bar{k}_{i} \tilde{y}_{i}\right) \leq 0 \text {. }
$$

Denote $\underline{K}=\operatorname{diag}\left(\underline{k}_{1}, \underline{k}_{2}, \ldots, \underline{k}_{p}\right)$ and $\bar{K}=\operatorname{diag}\left(\bar{k}_{1}, \bar{k}_{2}, \ldots, \bar{k}_{p}\right)$. Hence, one derives from (8) that

$$
(\tilde{f}(\tilde{y})-\underline{K} \tilde{y})^{T}(\tilde{f}(\tilde{y})-\bar{K} \tilde{y}) \leq 0 .
$$

Let $\phi(\tilde{y})=\tilde{f}(\tilde{y})-\underline{K} \tilde{y}$ and apply the loop transformation [41], the restriction (9) implies that $\phi(\tilde{y})$ satisfies

$$
\phi^{T}(\tilde{y})[\phi(\tilde{y})-(\bar{K}-\underline{K}) \tilde{y}] \leq 0 .
$$

Thus, system (7) can be written as

$$
\left\{\begin{array}{l}
\dot{\tilde{x}}(t)=(A+D \underline{K} C) \tilde{x}(t)+B \tilde{u}(t)+D \phi(\tilde{y}(t))+E \tilde{\omega}(t) \\
\tilde{y}(t)=C \tilde{x}(t)
\end{array}\right.
$$

where $\phi(\tilde{y})$ satisfies (10), that is, $\phi(\tilde{y})$ belongs to the sector $[0, \bar{K}-\underline{K}]$.

In order to eliminate the steady-state tracking error, it is desired to introduce the integral control action. For this purpose, we introduce the following integrator:

$$
\dot{q}(t)=e(t) .
$$

If the closed-loop system is asymptotically stable, $q(t)$ will tend toward a constant $q(\infty)$ asymptotically, namely $\lim _{t \rightarrow \infty} q(t)=q(\infty)$. Define $\tilde{q}(t)=q(t)-q(\infty)$, then from (12) we derive that $\tilde{q}(t)$ satisfies the dynamic equation:

$$
\dot{\tilde{q}}(t)=e(t)=C \tilde{x}(t)-\tilde{r}(t) .
$$

Combining (11) and (13), the following augmented system is obtained:

$$
\begin{aligned}
{\left[\begin{array}{c}
\dot{\tilde{q}}(t) \\
\dot{\tilde{x}}(t)
\end{array}\right]=} & {\left[\begin{array}{cc}
0 & C \\
0 & A+D \underline{K C}
\end{array}\right]\left[\begin{array}{c}
\tilde{q}(t) \\
\tilde{x}(t)
\end{array}\right]+\left[\begin{array}{c}
0 \\
B
\end{array}\right] \tilde{u}(t) } \\
& +\left[\begin{array}{l}
0 \\
D
\end{array}\right] \phi(\tilde{\tilde{y}}(t))+\left[\begin{array}{c}
-I \\
0
\end{array}\right] \tilde{r}(t)+\left[\begin{array}{l}
0 \\
E
\end{array}\right] \tilde{\omega}(t) .
\end{aligned}
$$

It is important to note that, as for the reference signal $r(t)$ at current time $t$, the future reference values $r(\tau)\left(t \leq \tau \leq t+l_{r}\right)$ are known in advance. We now attempt to make some necessary and mild changes to the structure of system (14), such that this useful future reference information can be effectively used in the tracking control structure and the original relationship of the system keeps unchanged. For this purpose, system (14) is re- 
formulated by

$$
\begin{aligned}
{\left[\begin{array}{c}
\dot{\tilde{q}}(t)-\left(r\left(t+l_{r}\right)-r(t)\right) \\
\dot{\tilde{x}}(t)
\end{array}\right]=} & {\left[\begin{array}{cc}
0 & C \\
0 & A+D \underline{K C}
\end{array}\right]\left[\begin{array}{c}
\tilde{q}(t) \\
\tilde{x}(t)
\end{array}\right]+\left[\begin{array}{l}
0 \\
B
\end{array}\right] \tilde{u}(t)+\left[\begin{array}{l}
0 \\
D
\end{array}\right] \phi(\tilde{y}(t)) } \\
& +\left[\begin{array}{c}
-I \\
0
\end{array}\right] \tilde{r}\left(t+l_{r}\right)+\left[\begin{array}{l}
0 \\
E
\end{array}\right] \tilde{\omega}(t) .
\end{aligned}
$$

It can be observed that the $(1,1)$-block and $(2,1)$-block of the state matrix in Eq. (15) are zeros, then in the light of the special structure of the state matrix, (15) can be rewritten as

$$
\begin{aligned}
{\left[\begin{array}{c}
\dot{\tilde{q}}(t)-\left(r\left(t+l_{r}\right)-r(t)\right) \\
\dot{\tilde{x}}(t)
\end{array}\right]=} & {\left[\begin{array}{cc}
0 & C \\
0 & A+D \underline{K C}
\end{array}\right]\left[\begin{array}{c}
\tilde{q}(t)-\int_{t}^{t+l_{r}} r(s) d s \\
\tilde{x}(t)
\end{array}\right]+\left[\begin{array}{l}
0 \\
B
\end{array}\right] \tilde{u}(t) } \\
& +\left[\begin{array}{c}
0 \\
D
\end{array}\right] \phi(\tilde{y}(t))+\left[\begin{array}{cc}
0 & -I \\
E & 0
\end{array}\right]\left[\begin{array}{c}
\tilde{\omega}(t) \\
\tilde{r}\left(t+l_{r}\right)
\end{array}\right] .
\end{aligned}
$$

Define a new state vector $\bar{x}(t)=\left[\left(\tilde{q}(t)-\int_{t}^{t+l_{r}} r(s) d s\right)^{T} \tilde{x}^{T}(t)\right]^{T}$ and a new disturbance vector $\bar{\omega}(t)=\left[\tilde{\omega}^{T}(t) \tilde{r}^{T}\left(t+l_{r}\right)\right]^{T} \in L_{2}$, then Eq. (16) is expressed by

$$
\dot{\bar{x}}(t)=\bar{A} \bar{x}(t)+\bar{B} \tilde{u}(t)+\bar{D} \phi(\tilde{y}(t))+\bar{E} \bar{\omega}(t),
$$

where

$$
\bar{A}=\left[\begin{array}{cc}
0 & C \\
0 & A+D \underline{K C}
\end{array}\right], \quad \bar{B}=\left[\begin{array}{l}
0 \\
B
\end{array}\right], \quad \bar{D}=\left[\begin{array}{l}
0 \\
D
\end{array}\right], \quad \bar{E}=\left[\begin{array}{cc}
0 & -I \\
E & 0
\end{array}\right] .
$$

Additionally, the output signal satisfies

$$
\tilde{y}(t)=C \tilde{x}(t)=\bar{C} \bar{x}(t)
$$

where $\bar{C}=\left[\begin{array}{ll}0 & C\end{array}\right]$.

As for system (17), we introduce the linear quadratic performance index:

$$
\begin{aligned}
J= & \int_{0}^{\infty}\left[\left(\tilde{q}(t)-\int_{t}^{t+l_{r}} r(s) d s\right)^{T} Q_{q}\left(\tilde{q}(t)-\int_{t}^{t+l_{r}} r(s) d s\right)\right. \\
& \left.+\tilde{x}^{T}(t) Q_{x} \tilde{x}(t)+\tilde{u}^{T}(t) R \tilde{u}(t)\right] d t \\
= & \int_{0}^{\infty}\left[\bar{x}^{T}(t) \bar{Q} \bar{x}(t)+\tilde{u}^{T}(t) R \tilde{u}(t)\right] d t,
\end{aligned}
$$

where $\bar{Q}=\operatorname{diag}\left(Q_{q}, Q_{x}\right)>0$ and $R>0$ are given weighting matrices with appropriate dimensions. Moreover, in the performance function $J$, the first term, $\int_{0}^{\infty}(\tilde{q}(t)-$ $\left.\left.\int_{t}^{t+l_{r}} r(s) d s\right)^{T} Q_{q}\left(\tilde{q}(t)-\int_{t}^{t+l_{r}} r(s) d s\right)\right) d t$, reflects the requirement of cumulative tracking error, the second one, $\int_{0}^{\infty} \tilde{x}^{T}(t) Q_{x} \tilde{x}(t) d t$, is the requirement of state deviation from the steady-state value, and the third one, $\int_{0}^{\infty} \tilde{u}^{T}(t) R \tilde{u}(t) d t$, is the restriction of the control range. Thus, the physical meaning is clear. This idea is similar to the conventional LQR 
control where the tracking error, the translated state and the control signal are tradeoff through adjusting the values of the weighting matrices $Q_{q}, Q_{x}$ and $R$.

Now, our task is to design a controller with preview action to make the system output $y(t)$ realize the asymptotic tracking of the reference signal $r(t)$, while minimizing the performance index $J$ in (18) as much as possible.

In fact, the performance index $J$ in (18) can also be expressed as a square of 2-norm of the following performance signal to be controlled:

$$
z(t)=M \bar{x}(t)+N \tilde{u}(t)
$$

where

$$
M=\left[\begin{array}{cc}
Q_{q}^{1 / 2} & 0 \\
0 & Q_{x}^{1 / 2} \\
0 & 0
\end{array}\right], \quad N=\left[\begin{array}{c}
0 \\
0 \\
R^{1 / 2}
\end{array}\right]
$$

That is,

$$
J=\int_{0}^{\infty} z^{T}(t) z(t) d t=\|z(t)\|_{2}^{2}
$$

Combining (17) and (19) leads to

$$
\left\{\begin{array}{l}
\dot{\bar{x}}(t)=\bar{A} \bar{x}(t)+\bar{B} \tilde{u}(t)+\bar{D} \phi(\tilde{y}(t))+\bar{E} \bar{\omega}(t), \\
\tilde{y}(t)=\bar{C} \bar{x}(t), \\
z(t)=M \bar{x}(t)+N \tilde{u}(t) .
\end{array}\right.
$$

It should be mentioned that the disturbance signal $\bar{\omega}(t)$ in $(20)$ is a signal with finite energy and the performance function (18) can be expressed by the square of the 2-norm of the performance signal $z(t)$. As a result, the classical $H_{\infty}$ control principle [37] can be employed to inhibit the undesirable effect of the disturbance signal $\bar{\omega}(t)$ on the performance signal $z(t)$. More specifically, $H_{\infty}$ control problem is to find a suitable controller $\tilde{u}(t)$ such that: (i) the closed-loop system of $(20)$ with $\bar{\omega}(t)=0$ is globally asymptotically stable; (ii) under the zero initial condition

$$
\|z(t)\|_{2} \leq \gamma\|\bar{\omega}(t)\|_{2}
$$

holds for arbitrary nonzero $\bar{\omega}(t) \in L_{2}$, where $\gamma>0$ is the disturbance attenuation level.

It is known that, in some practical problems, too large performance index may bring about high cost requirements. It can be observed from (21) and the relation between performance index $J$ and performance signal $z(t)$ that the smaller value $\gamma$ leads to the smaller value $J$. Therefore, in order to avoid the high cost, we could choose a smaller value $\gamma$ appropriately based on actual demand.

Remark 4 It is noteworthy that the popular Riccati equation approach in [8-13] cannot be directly used in this paper, since the system (1) under consideration is nonlinear and the 
uncertain nonlinearity belongs to a sector domain. Moreover, the conventional differentiation approach in [9-11] also fails to work in the derivation of error system due to the fact that the sector nonlinearity may be nondifferentiable. To overcome these obstacles, for the first time, we introduce a novel translation approach related to steady-state values and combine the state augmentation technique along with a reconstruction manner to derive the required augmented error system (17) including integrator and preview information. As a result, the original trajectory tracking problem is successfully converted into a standard $H_{\infty}$ control problem. Therefore, from the perspective of the construction of preview control system, our proposed approach is innovative.

\section{Design of the trajectory tracking controller with preview action}

For system (20), we consider the state feedback controller

$$
\tilde{u}(t)=K \bar{x}(t)
$$

where $K$ is the controller gain matrix to be determined. Substituting this controller into (20) results in the following closed-loop system:

$$
\left\{\begin{array}{l}
\dot{\bar{x}}(t)=(\bar{A}+\bar{B} K) \bar{x}(t)+\bar{D} \phi(\tilde{y}(t))+\bar{E} \bar{\omega}(t), \\
\tilde{y}(t)=\bar{C} \bar{x}(t), \\
z(t)=(M+N K) \bar{x}(t),
\end{array}\right.
$$

where $\phi(\tilde{y}(t))$ satisfies the sector condition (10).

To facilitate the presentation, we denote

$$
K_{\Delta}=\operatorname{diag}\left(\frac{\bar{k}_{1}-\underline{k}_{1}}{2}, \frac{\bar{k}_{2}-\underline{k}_{2}}{2}, \ldots, \frac{\bar{k}_{p}-\underline{k}_{p}}{2}\right), \quad Z=\operatorname{diag}\left(\mu_{1}, \mu_{2}, \ldots, \mu_{p}\right)
$$

Theorem 1 Suppose Assumptions A1-A4 are satisfied. The closed-loop system (23) is globally asymptotically stable and (21) holds for any non-zero $\bar{\omega}(t) \in L_{2}$ iffor given matrix $Z>0$ and the disturbance attenuation level $\gamma>0$ there exist matrices $P>0$ and $L$ such that

$$
\left[\begin{array}{cccc}
\bar{A} P+P \bar{A}^{T}+\bar{B} L+L^{T} \bar{B}^{T} & \bar{D}+P \bar{C}^{T} Z K_{\Delta} & \bar{E} & P M^{T}+L^{T} N^{T} \\
\bar{D}^{T}+K_{\Delta}{ }^{T} Z^{T} \bar{C} P & -Z & 0 & 0 \\
\bar{E}^{T} & 0 & -\gamma^{2} I & 0 \\
M P+N L & 0 & 0 & -I
\end{array}\right]<0 .
$$

Furthermore, the controller gain matrix is determined by $K=L P^{-1}$.

Proof The proof is divided into two parts. First, the global asymptotic stability of system (23) with $\bar{\omega}(t)=0$ is proved. Second, we shall show that (21) holds for all nonzero $\bar{\omega}(t)$ under zero initial condition.

(i) Consider the system (23) without disturbance, namely,

$$
\dot{\bar{x}}(t)=(\bar{A}+\bar{B} K) \bar{x}(t)+\bar{D} \phi(\tilde{y}(t)) .
$$


The Lyapunov function is selected as

$$
V(\bar{x})=\bar{x}^{T} P^{-1} \bar{x}
$$

Clearly, $V$ is positive definite due to $P^{-1}>0$. Taking the time-derivative of $V(\bar{x})$ along the trajectory of system (25), we deduce that

$$
\dot{V}(\bar{x})=\bar{x}^{T}(t)\left[P^{-1}(\bar{A}+\bar{B} K)+(\bar{A}+\bar{B} K)^{T} P^{-1}\right] \bar{x}(t)+2 \bar{x}^{T}(t) P^{-1} \bar{D} \phi(\tilde{y}(t)) .
$$

For the matrix $Z=\operatorname{diag}\left(\mu_{1}, \mu_{2}, \ldots, \mu_{p}\right)>0$, by the sector condition (10), one gets

$$
\phi(\tilde{y})^{T} Z \phi(\tilde{y})-2 \tilde{y}^{T} Z K_{\Delta} \phi(\tilde{y}) \leq 0 .
$$

Thus, the time-derivative of $V(\bar{x})$ along the trajectory of system (25) can be estimated by

$$
\begin{aligned}
& \dot{V}(\bar{x}) \leq \bar{x}^{T}(t)\left[P^{-1}(\bar{A}+\bar{B} K)+(\bar{A}+\bar{B} K)^{T} P^{-1}\right] \bar{x}(t)+2 \bar{x}^{T}(t) P^{-1} \bar{D} \phi(\tilde{y}(t)) \\
& -\phi^{T}(\tilde{y}) Z \phi(\tilde{y})+2 \tilde{y}^{T} Z K_{\Delta} \phi(\tilde{y}) \\
& =\left[\begin{array}{ll}
\bar{x}^{T}(t) & \phi^{T}(\tilde{y})
\end{array}\right] \Xi\left[\begin{array}{ll}
\bar{x}^{T}(t) & \phi^{T}(\tilde{y})
\end{array}\right]^{T},
\end{aligned}
$$

where

$$
\Xi=\left[\begin{array}{cc}
P^{-1}(\bar{A}+\bar{B} K)+(\bar{A}+\bar{B} K)^{T} P^{-1} & P^{-1} \bar{D}+\bar{C}^{T} Z K_{\Delta} \\
\bar{D}^{T} P^{-1}+K_{\Delta} Z \bar{C} & -Z
\end{array}\right] .
$$

It immediately follows from condition (24) that $\Xi<0$. Thus, $\dot{V}(\bar{x})$ is negative definite for all $\phi(\tilde{y})$ satisfying (10). Moreover, $V(\bar{x})$ is radially unbounded, that is, $V(\bar{x}) \rightarrow \infty$ as $\|x\| \rightarrow \infty$. Based on the Lyapunov stability theorem, system (25) is globally asymptotically stable.

(ii) When the disturbance $\bar{\omega}(t) \neq 0$, we still consider Lyapunov function (26). It is noted that, under the zero initial conditions (i.e. $\bar{x}(0)=0),\left.V(\bar{x}(t))\right|_{t=0}=0$ and $V(\bar{x}(t)) \geq 0$ for $t>0$. Define

$$
J_{0}=\int_{0}^{\infty}\left[z^{T}(t) z(t)-\gamma^{2} \bar{\omega}^{T}(t) \bar{\omega}(t)\right] d t
$$

Then we obtain

$$
\begin{aligned}
J_{0} & =\int_{0}^{\infty}\left[z^{T}(t) z(t)-\gamma^{2} \bar{\omega}^{T}(t) \bar{\omega}(t)+\dot{V}(\bar{x}(t))\right] d t+\left.V(\bar{x}(t))\right|_{t=0}-\left.V(\bar{x}(t))\right|_{t \rightarrow \infty} . \\
& \leq \int_{0}^{\infty}\left[z^{T}(t) z(t)-\gamma^{2} \bar{\omega}^{T}(t) \bar{\omega}(t)+\dot{V}(\bar{x}(t))\right] d t
\end{aligned}
$$

By some mathematical operations, one gets

$$
\begin{aligned}
& z^{T}(t) z(t)-\gamma^{2} \bar{\omega}^{T}(t) \bar{\omega}(t)+\dot{V}(\bar{x}(t)) \\
& \quad \leq \bar{x}^{T}(t)(M+N K)^{T}(M+N K) \bar{x}(t)-\gamma^{2} \bar{\omega}^{T}(t) \bar{\omega}(t)
\end{aligned}
$$




$$
\begin{aligned}
& +\bar{x}^{T}(t)\left[P^{-1}(\bar{A}+\bar{B} K)+(\bar{A}+\bar{B} K)^{T} P^{-1}\right] \bar{x}(t)+2 \bar{x}^{T}(t) P^{-1} \bar{D} \phi(\tilde{y}(t)) \\
& -\phi^{T}(\tilde{y}) Z \phi(\tilde{y})+2 \tilde{y}^{T} Z K_{\Delta} \phi(\tilde{y}) \\
& =\left[\begin{array}{lll}
\bar{x}^{T}(t) & \phi^{T}(\tilde{y}) & \bar{\omega}^{T}(t)
\end{array}\right] \Pi\left[\begin{array}{lll}
\bar{x}^{T}(t) & \phi^{T}(\tilde{y}) & \bar{\omega}^{T}(t)
\end{array}\right]^{T},
\end{aligned}
$$

where

$$
\Pi=\left[\begin{array}{ccc}
(M+N K)^{T}(M+N K)+P^{-1}(\bar{A}+\bar{B} K)+(\bar{A}+\bar{B} K)^{T} P^{-1} & P^{-1} \bar{D}+\bar{C}^{T} Z K_{\Delta} & P^{-1} \bar{E} \\
\bar{D}^{T} P^{-1}+K_{\Delta} Z \bar{C} & -Z & 0 \\
\bar{E}^{T} P^{-1} & 0 & -\gamma^{2} I
\end{array}\right] .
$$

Indeed, $J_{0} \leq 0$, that is, $\|z(t)\|_{2} \leq \gamma\|\bar{\omega}(t)\|_{2}$ is ensured by $\Pi<0$. Consequently, Eq. (21) holds. According to the Schur complement lemma (Lemma 1 ), $\Pi<0$ is equivalent to

$$
\left[\begin{array}{cccc}
P^{-1}(\bar{A}+\bar{B} K)+(\bar{A}+\bar{B} K)^{T} P^{-1} & P^{-1} \bar{D}+\bar{C}^{T} Z K_{\Delta} & P^{-1} \bar{E} & (M+N K)^{T} \\
\bar{D}^{T} P^{-1}+K_{\Delta} Z \bar{C} & -Z & 0 & 0 \\
\bar{E}^{T} P^{-1} & 0 & -\gamma^{2} I & 0 \\
M+N K & 0 & 0 & -I
\end{array}\right]<0 .
$$

By pre- and postmultiplying the left-side matrix in the above inequality, respectively, by $\operatorname{diag}(P, I, I)$ and its transpose, and denoting $L=K P$, condition (24) is derived instantly. Also, the controller gain matrix is determined by $K=L P^{-1}$. This completes the proof of Theorem 1.

Remark 5 If $\gamma>0$ is set to be a fixed parameter in advance, then by solving LMI (24) in Theorem 1 , a trajectory tracking controller satisfying a certain $H_{\infty}$ disturbance attenuation performance can be obtained. On the other hand, for given weighting matrices $Q_{q}$, $Q_{x}$ and $R$, a smaller performance level $\gamma$ means that the performance output $z(t)$ (a combination of the integral of the tracking error, the translated state vector and the control signal) is also smaller. Thus, it is of great importance to minimize the performance level $\lambda$. For this purpose, denote $\lambda=\gamma^{2}$ and solve the following optimization problem:

$$
\left\{\begin{array}{l}
\text { minimize } \lambda \\
\text { subject to } P>0, L \text { and LMI (24). }
\end{array}\right.
$$

If the above problem has a solution $(P, L, \lambda)$, then the optimal $H_{\infty}$ performance level is given by $\gamma^{*}=\sqrt{\lambda}$.

If the LMI condition (24) in Theorem 1 has a feasible solution $(P, L)$, then the controller ensuring the stability of the closed-loop system is given by Eq. (22) with the gain matrix $K=L P^{-1}$. For the purpose of clarifying the tracking controller structure, the gain matrix $K$ is partitioned as

$$
K=\left[\begin{array}{ll}
K_{\mathrm{er}} & K_{x}
\end{array}\right]
$$


where $K_{\mathrm{er}} \in R^{m \times p}$ and $K_{x} \in R^{m \times n}$. Accordingly, the control law (22) can be explicitly be written as follows:

$$
\tilde{u}(t)=K \bar{x}(t)=K_{\mathrm{er}}\left[\tilde{q}(t)-\int_{t}^{t+l_{r}} r(s) d s\right]+K_{x} \tilde{x}(t) .
$$

By the definition (6), it follows that

$$
\begin{aligned}
u(t) & =K_{\mathrm{er}}\left[\tilde{q}(t)-\int_{t}^{t+l_{r}} r(s) d s\right]+K_{x} \tilde{x}(t)+u(\infty) \\
& =K_{\mathrm{er}}[q(t)-q(\infty)]-K_{\mathrm{er}} \int_{t}^{t+l_{r}} r(s) d s+K_{x}[x(t)-x(\infty)]+u(\infty) .
\end{aligned}
$$

Based on the above analysis, the main result of this paper is summarized in the following theorem.

Theorem 2 Suppose Assumptions A1-A4 are satisfied. If the LMI feasibility problem (24) given in Theorem 1 is solvable, then the trajectory tracking controller with preview action for system (1) is

$$
u(t)=K_{x} x(t)+K_{\mathrm{er}} q(t)-K_{\mathrm{er}} \int_{t}^{t+l_{r}} r(s) d s+\left[-K_{x} x(\infty)-K_{\mathrm{er}} q(\infty)+u(\infty)\right],
$$

where the controller gain matrices $K_{\mathrm{er}}$ and $K_{x}$ are determined by (27). Under this controller, the output vector $y(t)$ can track the reference signal $r(t)$ asymptotically.

Remark 6 In this paper, a novel trajectory tracking scheme is proposed for continuoustime nonlinear Lur'e-type systems. From (29), it can be seen that the resulting control scheme consists of four parts. The first part $K_{x} x(t)$ represents the state feedback control action, the second part $K_{\mathrm{er}} q(t)=K_{\mathrm{er}}\left[\int_{0}^{t} e(s) d s+q(0)\right]$ represents the integral action which helps to eliminate the steady-state tracking error, i.e. integrator, the third one $-K_{\text {er }} \int_{t}^{t+l_{r}} r(s) d s$ represents the preview action based on the future reference trajectory used to improve the tracking performance, and the rest part depends on the steady-state values of the system. Here, $x(\infty)$ and $u(\infty)$ are computed by Eq. (5), while $q(\infty)$ is an ideal constant given by the designer representing the limit of error integral. Such a tracking control scheme is quite different from control strategies provided in [30-36, 42] where the future information about the predefined reference signal is not taken into consideration. In addition, by combining with the methodologies in [43-46], the preview tracking control for fractional nonlinear systems is an interesting research topic, which will be explored in future work.

\section{Numerical simulation}

In this section, an example is provided to illustrate the effectiveness and applicability of the proposed control scheme.

Example Consider system (1) with the following parameters:

$$
A=\left[\begin{array}{ll}
3 & -1 \\
1 & -1
\end{array}\right], \quad B=\left[\begin{array}{c}
10 \\
0
\end{array}\right], \quad C=\left[\begin{array}{ll}
1 & 0
\end{array}\right], \quad D=\left[\begin{array}{l}
1 \\
1
\end{array}\right], \quad E=\left[\begin{array}{l}
1 \\
0
\end{array}\right] .
$$


The nonlinear function $f(y)=0.5(|y+1|-|y-1|)$ satisfies Assumption A1 with $\underline{k}=0$ and $\bar{k}=1$. The external disturbance fulfilling A2 is chosen as $\omega(t)=1+e^{-2.5 t}$. Meanwhile, Assumption A3 is also fulfilled due to $\operatorname{rank}\left[\begin{array}{ll}A & B \\ C & 0\end{array}\right]=3$.

Fix $\gamma=1.5$ and set $Q_{e}=1.5, Q_{x}=0.1, R=0.1, Z=1$. With the aid of the Matlab LMI toolbox, LMI condition (24) returns the following solutions:

$$
P=\left[\begin{array}{ccc}
0.7929 & -1.5552 & 0.3185 \\
-1.5552 & 8.7954 & -0.4552 \\
0.3185 & -0.4552 & 10.4843
\end{array}\right], \quad L=\left[\begin{array}{lll}
0.3136 & -7.1384 & -0.0083
\end{array}\right]
$$

and then the controller gain matrix $K=L P^{-1}$ is derived. Through a suitable matrix partition, we get

$$
K_{\mathrm{er}}=-1.8344, \quad K_{x}=\left[\begin{array}{ll}
-1.1357 & 0.0056
\end{array}\right]
$$

In the light of Theorem 2, using the controller (29), the system output asymptotically tracks the reference signal. We now study the effect of the preview length $l_{r}$ on the trajectory tracking performance. For this purpose, the three situations $l_{r}=0, l_{r}=0.25$ and $l_{r}=0.5$ are taken into account. In what follows, the numerical simulation of the proposed control scheme is highlighted with two reference signals.

Case 1. The reference signal is taken as

$$
r(t)= \begin{cases}0, & t<5 \\ 3, & t \geq 5\end{cases}
$$

The output response of the closed-loop system, the tracking error, the control input and the state response are illustrated in Figs. 1-4, respectively.

We can see from Fig. 1 that the controllers designed in three cases can guarantee that the output of the closed-loop system accurately tracks the reference signal. Compared to the traditional method without preview (i.e. $l_{r}=0$ ), the proposed preview control method makes the closed-loop system respond faster and reach the desired reference value earlier. The detailed transient characteristics (delay time, rise time and settling time) are presented in Table 1, from which we can see that the transient performance indices decrease

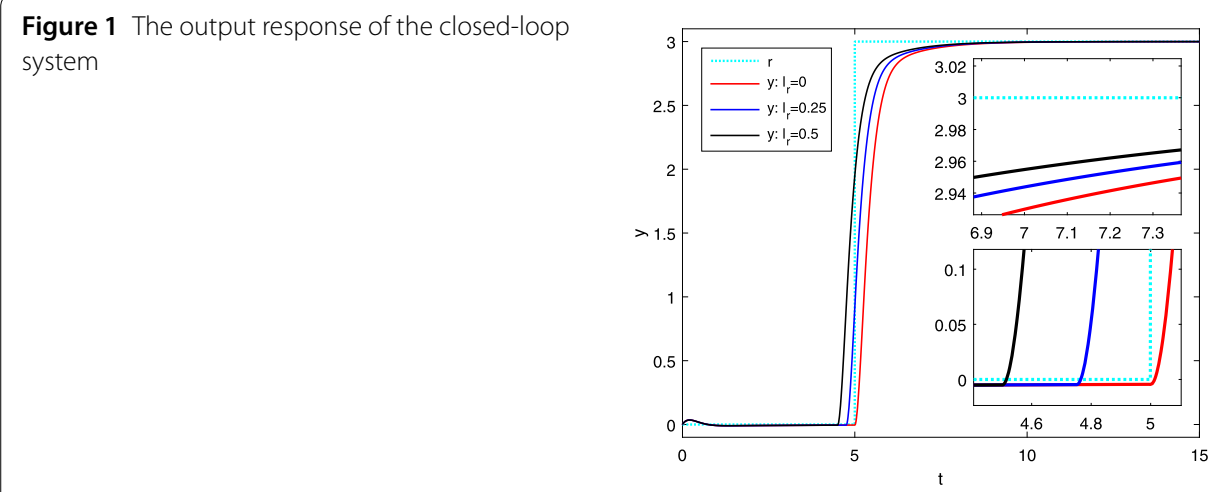


Figure 2 The tracking error

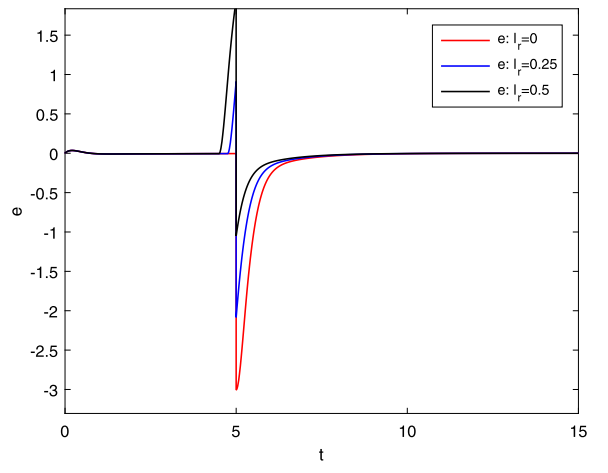

Figure 3 The control input

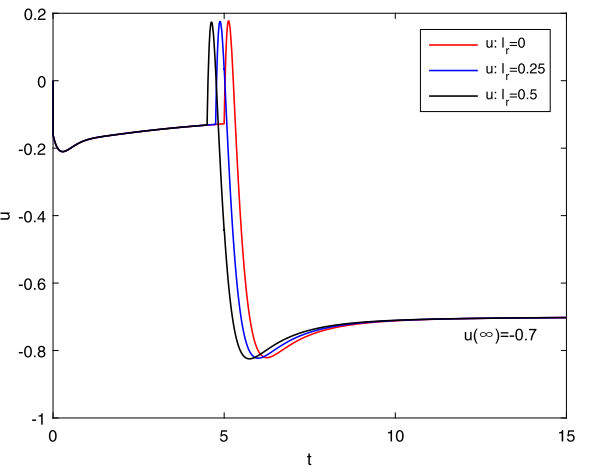

Figure 4 The state response

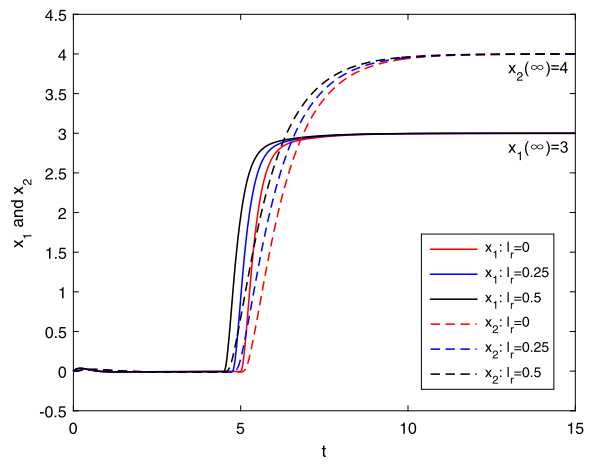

Table 1 Transient tracking performance index results

\begin{tabular}{llll}
\hline Index & $\begin{array}{l}\text { Traditional method } \\
\left(I_{r}=0\right)\end{array}$ & $\begin{array}{l}\text { Proposed method } \\
\left(I_{r}=0.25\right)\end{array}$ & $\begin{array}{l}\text { Proposed method } \\
\left(I_{r}=0.5\right)\end{array}$ \\
\hline Delay time & 5.3742 & 5.1241 & 4.8740 \\
Rise time & 0.8342 & 0.8329 & 0.8317 \\
Settling time & 7.1740 & 6.9282 & 6.6756 \\
\hline
\end{tabular}

as the preview length increases. In summary, the closed-loop system exhibits good dynamic response and high steady-state accuracy due to the use of preview information.

Moreover, Fig. 2 demonstrates that the preview of reference signal is quite helpful to improve the tracking accuracy of the closed-loop system. As shown in Fig. 3, it is reason- 
able that the suggested control signal varies within a bounded range. With the appropriate increment of the preview length, the control signal changes in advance, which also reflects an inherent characteristic of the preview control principle. In addition, it can be computed from (5) that the steady-state values in this example are $x(\infty)=\left[\begin{array}{l}3 \\ 4\end{array}\right]$ and $u(\infty)=-0.7$. As Figs. 3 and 4 show, the control signal and state vector tend toward their steady-state values ultimately, which confirms the previous theoretical results.

Case 2. The reference signal is taken as

$$
r(t)= \begin{cases}0, & t<5 \\ 0.6(t-5), & 5 \leq t \leq 10 \\ 3, & t>10\end{cases}
$$

The output response of the closed-loop system, the tracking error between the actual output and the desired reference signal, the control input and the state response are shown in Figs. 5-8, respectively.

From Figs. 5 and 6, we see that the traditional controller without preview action leads to large error during the output tracking process, while the preview controller could substantially reduce the output error and improve the tracking precision of the control system. Moreover, the detailed transient characteristics (delay time, rise time and settling time) are presented in Table 2, where the transient performance indices decreases with the increase of the preview length. Furthermore, as shown in Figs. 7 and 8, the control input

Figure 5 The output response of the closed-loop system

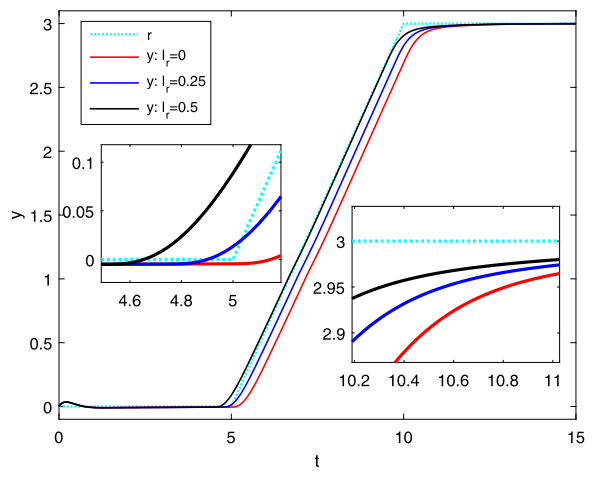

Figure 6 The tracking error

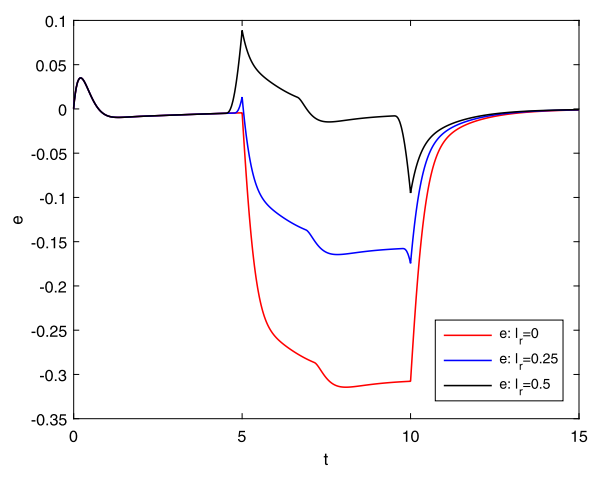


Figure 7 The control input

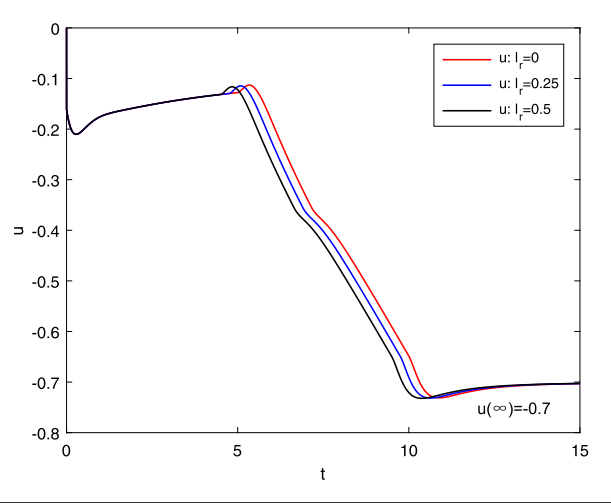

Figure 8 The state response

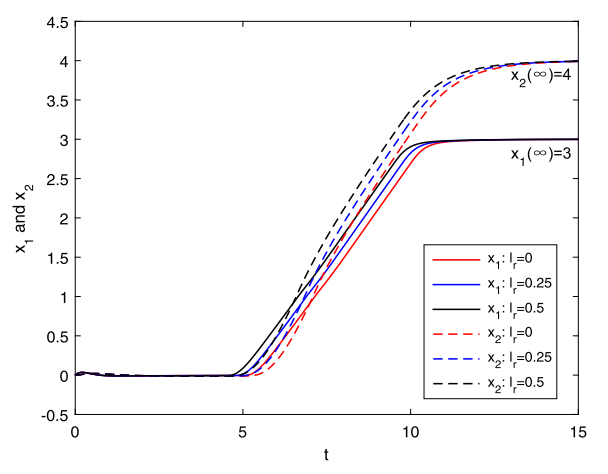

Table 2 Transient tracking performance index results

\begin{tabular}{lccc}
\hline Index & Traditional method & Proposed method & $\left(I_{r}=0.25\right)$ \\
& $\left(I_{r}=0\right)$ & 7.7728 & $\begin{array}{c}\text { Proposed method } \\
\left(I_{r}=0.5\right)\end{array}$ \\
\hline Delay time & 8.0261 & 4.0889 & 7.5258 \\
Rise time & 4.0891 & 10.4632 & 4.0877 \\
Settling time & 10.7130 & & 10.2100 \\
\hline
\end{tabular}

and state vectors tend to their steady-state values eventually, illustrating the effectiveness of the proposed method.

\section{Conclusions}

In this paper, the trajectory tracking problem based on preview control is studied for a class of continuous-time nonlinear Lur'e-type systems. First, the translation approach and the state augmentation are used to derive an error system. Then, with the aid of a reconstruction method and some special mathematical operations, the reference preview information is systematically included and the required augmented error system is successfully constructed. The trajectory tracking problem is thereby reduced into a standard $H_{\infty}$ control problem. Furthermore, the controller design condition is presented to ensure the overall stability and $H_{\infty}$ performance of the closed-loop system. Based on this, a trajectory tracking controller, which consists of the state feedback control action, the integral control action and the preview action, is obtained for the original system. Simulation results confirm the effectiveness and merits of the proposed control scheme. 


\section{Acknowledgements}

The authors sincerely thank the referees and the editors for their helpful comments and suggestions.

\section{Funding}

This work was supported by Doctoral Research Fund in Shandong Jianzhu University, National Natural Science Foundation of China (No. 61803228, 61903130) and Hubei Provincial Natural Science Foundation of China (No. 2019CFB227).

\section{Availability of data and materials}

Data sharing is not applicable to this article as no datasets were generated or analyzed during the current study.

\section{Ethics approval and consent to participate}

Not applicable.

\section{Competing interests}

The authors declare to have no potential conflicts of interest with respect to the research, authorship, and publication of this article.

\section{Consent for publication}

Not applicable.

\section{Authors' contributions}

All authors equally contributed in the preparation of this manuscript. All authors read and approved the final manuscript.

\section{Author details}

${ }^{1}$ School of Science, Shandong Jianzhu University, Jinan, China. ${ }^{2}$ School of Information Management and Statistics, Hubei University of Economics, Wuhan, China.

\section{Publisher's Note}

Springer Nature remains neutral with regard to jurisdictional claims in published maps and institutional affiliations.

\section{Received: 14 February 2020 Accepted: 4 June 2020 Published online: 15 June 2020}

\section{References}

1. Tsuchiya, T., Egami, T.: Digital Preview and Predictive Control (translated by Fucheng Liao). Beijing Science and Technology Press, Beijing (1994)

2. Birla, N., Swarup, A.: Optimal preview control: a review. Optim. Control Appl. Methods 36(2), 241-268 (2015)

3. Sheridan, T.B.: Three models of preview control. IEEE Trans. Hum. Factors Electron. HFE-7(2), 91-102 (1966)

4. Bender, E.K.: Optimum linear preview control with application to vehicle suspension. J. Basic Eng. 90(2), 213-221 (1968)

5. Hayase, M., Ichikawa, K.: Optimal servosystem utilizing future value of desired function. Trans. Soc. Instrum. Control Eng. 5(1), 86-94 (1969)

6. Tomizuka, M.: Optimal continuous finite preview problem. IEEE Trans. Autom. Control 20(3), 362-365 (1975)

7. Tomizuka, M., Rosenthal, D.E.: On the optimal digital state vector feedback controller with integral and preview actions. J. Dyn. Syst. Meas. Control 101(2), 172-178 (1979)

8. Katayama, T., Ohki, T., Inoue, T., Kato, T.: Design of an optimal controller for a discrete-time system subject to previewable demand. Int. J. Control 41(3), 677-699 (1985)

9. Katayama, T., Hirono, T.: Design of an optimal servomechanism with preview action and its dual problem. Int. J. Control 45(2), 407-420 (1987)

10. Liao, F., Tang, Y.Y., Liu, H., Wang, Y.: Design of an optimal preview controller for continuous-time systems. Int. J. Wavelets Multiresolut. Inf. Process. 9(4), 655-673 (2011)

11. Wu, J., Liao, F., Tomizuka, M.: Optimal preview control for a linear continuous-time stochastic control system in finite-time horizon. Int. J. Syst. Sci. 48(1), 129-137 (2017)

12. Zhang, W., Bae, J., Tomizuka, M.: Modified preview control for a wireless tracking control system with packet loss. IEEE/ASME Trans. Mechatron. 20(1), 299-307 (2015)

13. Tsai, J.S.-H., Liao, Y.-T., Ebrahimzadeh, F., Lai, S.-Y., Su, T.-J., Guo, S.-M., Shieh, L.-S., Tsai, T.-J.: A new Pl optimal linear quadratic state-estimate tracker for continuous-time non-square nonminimum phase systems. Int. J. Syst. Sci. 48(7), 1438-1459 (2017)

14. Zhen, Z., Wang, Z., Wang, D.: Information fusion estimation based preview control for discrete linear system. Acta Autom. Sin. 36(2), 347-352 (2010)

15. Zhen, Z., Wang, Z., Wang, D.: Optimal preview tracking control based on information fusion in error system. Control Theory Appl. 26(4), 425-428 (2009)

16. Wang, Z., Jiang, B., Zhen, Z.: Fusion Estimation and Fusion Control. Beijing Science Press, Beijing (2009)

17. Liao, F., Cao, M., Hu, Z., An, P.: Design of an optimal preview controller for linear discrete-time causal descriptor systems. Int. J. Control 85(10), 1616-1624 (2012)

18. Cao, M., Liao, F.: Design of an optimal preview controller for linear discrete-time descriptor systems with state delay. Int. J. Syst. Sci. 46(5), 932-943 (2015)

19. Lu, Y., Liao, F., Deng, J., Pattinson, C.: Cooperative optimal preview tracking for linear descriptor multi-agent systems J. Franklin Inst. 356(2), 908-934 (2019)

20. Zhao, L., Sun, F., Ren, J., Li, B.: Optimal preview control for a class of continuous time-invariant descriptor systems. Optim. Control Appl. Methods 37(2), 279-289 (2016) 
21. Liao, F., Ren, Z., Tomizuka, M.: Preview control for impulse-free continuous-time descriptor systems. Int. J. Control 88(6), 1142-1149 (2015)

22. Takaba, K.: Robust servomechanism with preview action for polytopic uncertain systems. Int. J. Robust Nonlinear Control 10(2), 101-111 (2000)

23. Li, L., Liao, F.: $H_{\infty}$ preview control of a class of uncertain discrete-time systems. Asian J. Control 19(5), 1-15 (2017)

24. Li, L., Liao, F.: Robust preview control for a class of uncertain discrete-time systems with time-varying delay. ISA Trans. 73, 11-21 (2018)

25. Yu, X., Liao, F., Deng, J.: Tracking controller design with preview action for a class of Lipschitz nonlinear systems and its applications. Circuits Syst. Signal Process. 39(6), 2922-2947 (2020)

26. Achnib, A., Airimitoaie, T.-B., Lanusse, P., Abrashov, S., Aoun, M., Chetoui, M.: Discrete-time robust control with an anticipative action for preview systems. J. Dyn. Syst. Meas. Control 141(3), 031012 (2019)

27. Lee, S.M., Park, J.H.: Robust stabilization of discrete-time nonlinear Lur'e systems with sector and slope restricted nonlinearities. Appl. Math. Comput. 200(1), 429-436 (2008)

28. Kim, K.K.K., Braatz, R.D.: Observer-based output feedback control of discrete-time Lur'e systems with sector-bounded and slope-restricted nonlinearities. Int. J. Robust Nonlinear Control 24(16), 2458-2472 (2014)

29. Grujic, L.T., Porter, B.: Continuous-time tracking systems incorporating Lur'e plants with single non-linearities. Int. J. Syst. Sci. 11(2), 177-189 (1980)

30. Porter, B., Jones, A.H.: Design of tunable digital set-point tracking and disturbance-rejection controllers for Lur'e plants with multiple non-linearities. Int. J. Syst. Sci. 15(5), 501-511 (1984)

31. Grujic, L.T.: Algebraic conditions for absolute tracking control of Lurie systems. Int. J. Control 48(2), 729-754 (1988)

32. Wang, H., Lin, Y., Xue, A., Pan, H., Lu, R.: Reliable robust $H_{\infty}$ tracking control for a class of uncertain Lur'e singular systems. Acta Autom. Sin. 34(8), 893-899 (2008)

33. Fedele, G., D'Aquila, G.: High-gain fractional-order controller for output tracking and disturbance attenuation in a class of Lur'e systems. IFAC-PapersOnLine 48(11), 748-753 (2015)

34. Lee, S.M., Kwon, O.M., Park, J.H.: Output feedback model predictive tracking control using a slope bounded nonlinear model. J. Optim. Theory Appl. 160, 239-254 (2014)

35. Zhao, Y., Duan, Z., Wen, G.: Robust consensus tracking of multi-agent systems with uncertain Lur'e-type non-linear dynamics. IET Control Theory Appl. 7(9), 1249-1260 (2013)

36. Liu, M., Li, Z.: Robust consensus of Lur'e networks with uncertain communications. IET Control Theory Appl. 11(6), 877-882 (2017)

37. Cao, J., Sivasamy, R., Rakkiyappan, R.: Sampled-data $H_{\infty}$ synchronization of chaotic Lur'e systems with time delay. Circuits Syst. Signal Process. 35(3), 811-835 (2016)

38. Huang, H., Feng, G., Cao, J.: Exponential synchronization of chaotic Lur'e systems with delayed feedback control. Nonlinear Dyn. 57, 441-453 (2009)

39. Zhang, H., Cao, J., Xiong, L.: Novel synchronization conditions for time-varying delayed Lur'e system with parametric uncertainty. Appl. Math. Comput. 350, 224-236 (2019)

40. DeLellis, P., Di Bernardo, M., Garofalo, F.: Adaptive pinning control of networks of circuits and systems in Lur'e form. IEEE Trans. Circuits Syst. I, Regul. Pap. 60(11), 3033-3042 (2013)

41. Khalil, H.K.: Nonlinear Systems. Prentice Hall, Upper Saddle River (1996)

42. Wang, C., Wu, Y., Yu, J.: Barrier Lyapunov functions-based dynamic surface control for pure-feedback systems with full state constraints. IET Control Theory Appl. 11(4), 524-530 (2017)

43. Shah, K., Ali, A., Bushnaq, S.: Hyers-Ulam stability analysis to implicit Cauchy problem of fractional differential equations with impulsive conditions. Math. Methods Appl. Sci. 41(17), 8329-8343 (2018)

44. Shah, K., Hussain, W. Investigating a class of nonlinear fractional differential equations and its Hyers-Ulam stability by means of topological degree theory. Numer. Funct. Anal. Optim. 40(12), 1355-1372 (2019)

45. Harikrishnan, S., Shah, K., Baleanu, D., Kanagarajan, K.: Note on the solution of random differential equations via $\psi$-Hilfer fractional derivative. Adv. Differ. Equ. 2018, 224 (2018)

46. Shah, K., Wang, J., Khalil, H., Khan, R.A.: Existence and numerical solutions of a coupled system of integral BVP for fractional differential equations. Adv. Differ. Equ. 2018, 149 (2018)

\section{Submit your manuscript to a SpringerOpen ${ }^{\circ}$ journal and benefit from:}

- Convenient online submission

- Rigorous peer review

- Open access: articles freely available online

- High visibility within the field

- Retaining the copyright to your article

Submit your next manuscript at $\gg$ springeropen.com 